\title{
Identification of Psychoactive Degradants of Cannabidiol in Simulated Gastric and Physiological Fluid
}

\author{
John Merrick, Brian Lane, ${ }^{1}$ Terri Sebree, ${ }^{2, *}$ Tony Yaksh, ${ }^{3}$ Carol O'Neill, ${ }^{2}$ and Stan L. Banks ${ }^{2}$
}

\begin{abstract}
Introduction: In recent research, orally administered cannabidiol (CBD) showed a relatively high incidence of somnolence in a pediatric population. Previous work has suggested that when CBD is exposed to an acidic environment, it degrades to $\Delta^{9}$-tetrahydrocannabinol $(T H C)$ and other psychoactive cannabinoids. To gain a better understanding of quantitative exposure, we completed an in vitro study by evaluating the formation of psychoactive cannabinoids when CBD is exposed to simulated gastric fluid (SGF).

Methods: Materials included synthetic CBD, $\Delta^{8}-\mathrm{THC}$, and $\Delta^{9}$-THC. Linearity was demonstrated for each component over the concentration range used in this study. CBD was spiked into media containing $1 \%$ sodium dodecyl sulfate (SDS). Samples were analyzed using chromatography with UV and mass spectrometry detection. An assessment time of $3 \mathrm{~h}$ was chosen as representative of the maximal duration of exposure to gastric fluid.

Results: CBD in SGF with 1\% SDS was degraded about $85 \%$ after 60 min and more than $98 \%$ at 120 min. The degradation followed first-order kinetics at a rate constant of $-0.031 \mathrm{~min}^{-1}\left(R^{2}=0.9933\right)$. The major products formed were $\Delta^{9}$-THC and $\Delta^{8}$-THC with less significant levels of other related cannabinoids. CBD in physiological buffer performed as a control did not convert to THC. Confirmation of THC formation was demonstrated by comparison of mass spectral analysis, mass identification, and retention time of $\Delta^{9}-\mathrm{THC}$ and $\Delta^{8}$-THC in the SGF samples against authentic reference standards.

Conclusions: SGF converts CBD into the psychoactive components $\Delta^{9}$-THC and $\Delta^{8}$-THC. The first-order kinetics observed in this study allowed estimated levels to be calculated and indicated that the acidic environment during normal gastrointestinal transit can expose orally CBD-treated patients to levels of THC and other psychoactive cannabinoids that may exceed the threshold for a physiological response. Delivery methods that decrease the potential for formation of psychoactive cannabinoids should be explored.
\end{abstract}

Key words: cannabidiol; degredation; drug discovery; gastric fluid; kinetics; THC

\section{Introduction}

The flowering plants of the genus Cannabis, which mainly comprises the sativa and indica species, ${ }^{1,2}$ have been recognized for medical treatment for millennia. Although Cannabis contains nearly 500 compounds from 18 chemical classes, its physiological effects derive mainly from a family of naturally occurring compounds known as plant cannabinoids or phytocannabinoids. Of the more than 100 phytocannabinoids that have been identified in Cannabis, ${ }^{3}$ among the most important and widely studied are its main psychoactive constituent, $\Delta^{9}$ tetrahydrocannabinol $\left(\Delta^{9}\right.$-THC $),{ }^{4}$ and the most important nonpsychoactive component, cannabidiol (CBD) ${ }^{5}$ Other biologically active phytocannabinoids that have been isolated in Cannabis include $\Delta^{8}$-THC, cannabinol, $\Delta^{9}$-tetrahydrocannabivarin, and cannabidivarin. ${ }^{2,6}$

\footnotetext{
${ }^{1}$ Pace Analytical Services, Oakdale, Minnesota.

${ }^{2}$ Zynerba Pharmaceuticals, Inc., Devon, Pennsylvania.

${ }^{3}$ Department of Anesthesiology, University of California, San Diego, La Jolla, California.

*Address correspondence to: Terri Sebree, BS, Zynerba Pharmaceuticals, Inc., 80 West Lancaster Avenue, Suite 300, Devon, PA 19333, E-mail: sebreet@zynerba.com
}

() John Merrick et al. 2016; Published by Mary Ann Liebert, Inc. This Open Access article is distributed under the terms of the Creative Commons License (http://creativecommons.org/licenses/by/4.0), which permits unrestricted use, distribution, and reproduction in any medium, provided the original work is properly credited. 
THC and CBD produce a wide range of pharmacological effects by interacting with an endogenous lipid-signaling network known as the endocannabinoid system, specifically with two G-protein-coupled receptors known as cannabinoid $1\left(\mathrm{CB}_{1}\right)$ and cannabinoid $2\left(\mathrm{CB}_{2}\right) .{ }^{7} \mathrm{CB}_{1}$ receptors are densely expressed in the brain $^{8}$ and they have been detected in dorsal primary afferent spinal cord regions and spinal interneurons, ${ }^{9-11}$ whereas $\mathrm{CB}_{2}$ receptors are located primarily in the tissues of the immune system (macrophages), as well as in non-neuronal cells, such as astrocytes, oligodendrocytes, and microglia. ${ }^{12}$ As a result of the wide distribution of these receptors, cannabinoids are thought to play a role in an array of physiological and pathophysiological processes. ${ }^{8,9,13,14}$

THC is considered a partial agonist at $\mathrm{CB}_{1}$ and $\mathrm{CB}_{2}$ receptors. ${ }^{5,7}$ Its activity at these receptors has been associated with numerous physiological effects, such as inhibiting adenylate cyclase activity and $\mathrm{Ca}^{2+}$ influx, decreasing formation of cyclic adenosine monophosphate and protein kinase A activity, activating inwardly rectifying potassium channels and stimulating the mitogenactivated protein kinase-signaling cascades. ${ }^{12,15} \mathrm{CBD}$ has an affinity for $\mathrm{CB}_{1}$ and $\mathrm{CB}_{2}$ receptors in the micromolar range ${ }^{5,7,16}$ and has been described as a noncompetitive inverse agonist (e.g., potentially inhibiting the activity of cannabinoid agonists). ${ }^{7}$ However, the pharmacodynamic profile of CBD includes a variety of effects, including blocking the equilibrative nucleoside transporter (ENT1) and enhancing functionality of the $5-\mathrm{HT}_{1 \mathrm{a}}$ receptor, glycine receptors, the transient receptor potential of vanilloid type- 1 channel, and the melastatin type 8 channel. ${ }^{7}$ In addition, CBD has been shown to regulate the intracellular effects of calcium and ligand binding to several receptors, including the G-proteincoupled receptor GPR55. ${ }^{2}$ Functionally, CBD can exert a range of anti-inflammatory effects, including attenua- tion of endothelial cell activation, chemotaxis of inflammatory cells, suppression of T-cell macrophage reactivity, and induction of apoptosis of $\mathrm{T}$ cells. ${ }^{2,7}$

Well-controlled studies have begun to clarify the therapeutic potential of the phytocannabinoids. With THC, for example, clinical and preclinical data support its ability to treat pain, reduce nausea and vomiting, and increase appetite. ${ }^{1,7,17} \mathrm{CBD}$ has shown antiemetic, anticonvulsant, anti-inflammatory, and antipsychotic properties in animal studies. ${ }^{18-21}$ Clinical trials have been conducted with a variety of disease states, among them multiple sclerosis, schizophrenia, bipolar mania, social anxiety disorder, insomnia, Huntington's disease, and epilepsy. ${ }^{16}$ Overall, CBD has a positive safety profile, ${ }^{22}$ and there have been encouraging results in the treatment of patients with inflammation, diabetes, cancer, affective disorders, neurodegenerative diseases, ${ }^{23}$ and epilepsy. ${ }^{2,18,24}$ These clinical studies with CBD, particularly in patients with epilepsy, have generated interest in its medical application and attracted attention in the popular media. ${ }^{25}$

In recent epilepsy research, pediatric subjects receiving orally administered CBD showed a relatively high incidence of adverse events ( $\leq 44 \%)$, with somnolence $(\leq 21 \%)$ and fatigue $(\leq 17 \%)$ among the most common. ${ }^{26,27}$ If CBD is nonpsychoactive, we wondered whether these responses might be associated with a clinical manifestation of findings from experimental work, ${ }^{28}$ suggesting that when CBD is degraded in an acidic environment, it rapidly cyclizes to $\Delta^{9}$-THC and other psychoactive cannabinoids. To test the hypothesis that CBD might be converted to THC in the acidic environment of the stomach (Fig. 1), an in vitro study was completed by evaluating the formation of psychoactive cannabinoids as possible degradation products of oral CBD under simulated gastric and physiological conditions. Due to the limited aqueous solubility of $\mathrm{CBD}$, an approach to
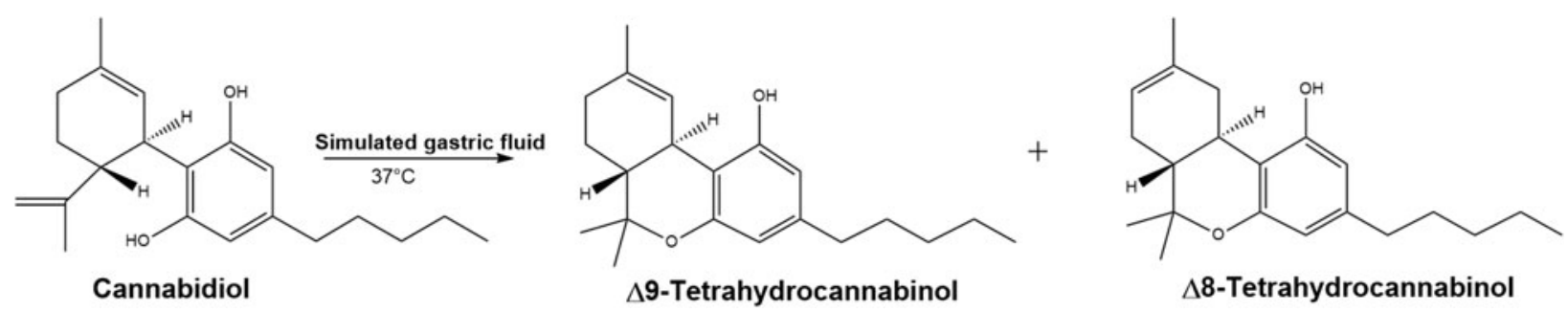

$\Delta 8$-Tetrahydrocannabinol

FIG. 1. Psychoactive products of acid-catalyzed cyclization of $\mathrm{CBD}$ in the presence of $\mathrm{SGF}$ at $37^{\circ} \mathrm{C}$. $\mathrm{CBD}$, cannabidiol; SGF, simulated gastric fluid. 
improve the solubility was determined. The approach recommended in United States Pharmacopeia (USP) ${ }^{29}$ to use a surfactant was implemented and it was found that $1 \%$ sodium dodecyl sulfate (SDS) was required. Samples from the study were assayed through ultraperformance liquid chromatography (UPLC) with UV and tandem mass spectroscopy detection (LC/MS/MS) to confirm the appropriate molecular weight. Reference standards for CBD, $\Delta^{8}$-THC, and $\Delta^{9}$-THC were used in this study.

\section{Materials and Methods}

Materials included synthetic CBD (99\% purity; Zynerba Pharmaceuticals, Lot: MG-24-156 R2-B), $\Delta^{8}$-THC (100\% purity; Cayman Chemicals, Lot: 0458238-2), and $\Delta^{9}$-THC (100\% purity; Cayman Chemicals, Lot: 0459902-3). Samples were analyzed on a Waters Acquity $\mathrm{H}$ Class UPLC system with UV detection followed by analysis through a Waters Acquity tandem quadrupole mass spectrometry (TQD MS/MS) detector. CBD stability studies in simulated gastric fluid (SGF) and physiological buffer [4-(2-hydroxyethyl)-1-piperazineethanesulfonic acid; HEPES] were performed in a USP Apparatus II dissolution bath (Distek model 2100B). UPLC and MS method parameters for the stability studies and sample analysis are listed in Tables 1 and 2, respectively. Conditions for the dissolution apparatus are listed in Table 3.

\section{UPLC with UV and MS/MS analyses}

of SDS-containing solutions

Linearity was assessed for CBD, $\Delta^{8}$-THC, and $\Delta^{9}$-THC by calculating the regression line, expressed by the coefficient of determination $\left(R^{2}\right)$, and evaluated from $\sim 0.1 \%$ to $120 \%$ of the target CBD sample concentration $(8.0 \mu \mathrm{g} / \mathrm{mL})$, which is equivalent to concentrations ranging from 0.008 to $9.6 \mu \mathrm{g} / \mathrm{mL}$ for each component. The UV response was used for quantification.

Table 1. Conditions and Set Points for the UltraPerformance Liquid Chromatography

\begin{tabular}{ll}
\hline Condition & \multicolumn{1}{c}{ Set point } \\
\hline Mobile phase A & $2 \mathrm{mM}$ ammonium formate, $\mathrm{pH} 4.8$ \\
Mobile phase B & Methanol \\
Mobile phase ratio & $30: 70 \mathrm{~A}: \mathrm{B}$ \\
Column & Waters HSS C18 $50 \times 2.1 \mathrm{~mm}, 1.8 \mu \mathrm{m}$ \\
Flow rate & $0.5 \mathrm{~mL} / \mathrm{min}$ \\
Wavelength & $222 \mathrm{~nm}$ \\
Column temperature & $50^{\circ} \mathrm{C}$ \\
Injection volume & $10 \mu \mathrm{L}$
\end{tabular}

HSS, high-strength silica.
Table 2. Settings for the Tandem Quadrupole Mass Spectrometry and Multiple Reaction Monitoring

\begin{tabular}{|c|c|c|c|c|c|}
\hline \multicolumn{6}{|c|}{ TQD MS/MS } \\
\hline \multicolumn{4}{|c|}{ lonization mode } & \multicolumn{2}{|c|}{ ESI positive } \\
\hline \multicolumn{4}{|c|}{ Capillary voltage } & \multicolumn{2}{|c|}{$3.5 \mathrm{kV}$} \\
\hline \multicolumn{4}{|c|}{ Cone voltage } & \multicolumn{2}{|c|}{$30-35 \mathrm{~V}$} \\
\hline \multicolumn{4}{|c|}{ Desolvation gas temperature } & \multicolumn{2}{|c|}{$\begin{array}{c}30-35 \mathrm{~V} \\
250^{\circ} \mathrm{C}\end{array}$} \\
\hline \multicolumn{4}{|c|}{ Desolvation gas flow } & \multicolumn{2}{|c|}{$500 \mathrm{~L} / \mathrm{h}$} \\
\hline \multicolumn{4}{|c|}{ Source temperature } & \multicolumn{2}{|c|}{$150^{\circ} \mathrm{C}$} \\
\hline \multicolumn{4}{|c|}{ Cone gas flow } & \multicolumn{2}{|c|}{$50 \mathrm{~L} / \mathrm{h}$} \\
\hline \multicolumn{4}{|c|}{ Mass scan range } & \multicolumn{2}{|c|}{$150-650 \mathrm{Da}$} \\
\hline \multicolumn{6}{|l|}{ MRM } \\
\hline $\begin{array}{l}\text { Precursor } \\
\text { ion }(\mathrm{m} / \mathrm{z})\end{array}$ & $\begin{array}{l}\text { Product } \\
\text { ion }(\mathrm{m} / \mathrm{z})\end{array}$ & $\begin{array}{c}\text { Dwell } \\
\text { time (sec) }\end{array}$ & $\begin{array}{c}\text { Cone } \\
\text { voltage (V) }\end{array}$ & $\begin{array}{l}\text { Collision } \\
\text { energy (V) }\end{array}$ & Use \\
\hline 315.2 & 193.2 & 0.200 & 35 & 20 & $\overline{\text { Quantifier }}$ \\
\hline 315.2 & 259.3 & 0.200 & 35 & 20 & Qualifier \\
\hline
\end{tabular}

ESI, electrospray ionization; MRM, multiple reaction monitoring; TQD MS/MS, tandem quadrupole mass spectrometry.

Incubation buffers and protocol

A stock solution of $\sim 40 \mathrm{mg} / \mathrm{mL}$ CBD was prepared in methanol. Using USP dissolution procedure guidance, ${ }^{29}$ $1 \%$ SDS was added to media to solubilize $1.0 \mathrm{~mL}$ of the $40 \mathrm{mg} / \mathrm{mL}$ CBD stock solution; the resulting incubation media contained $0.2 \%$ methanol. CBD-containing solutions were prepared in amber glassware, amber dissolution vessels were used in the dissolution bath, and all sampling was performed under ultraviolet-filtered yellow lighting to protect solutions from light. Incubation studies were carried out in two media: one SGF and one simulating a physiological buffer.

SGF with $1 \%$ SDS was prepared by adding $\sim 10 \mathrm{~g}$ of SDS (Ultra Pure; MP Chemicals, lot: M9655) to $1 \mathrm{~L}$ of SGF prepared from enzyme-free concentrate (Ricca; pepsin free lot: 4505367 ), equivalent to $0.1 \mathrm{M}$ as hydrochloric acid and $0.2 \%$ sodium chloride.

Physiological buffer with $1 \%$ SDS was prepared similarly by adding SDS to $1 \mathrm{~L}$ of HEPES buffer, $\mathrm{pH} 7.4$ (HEPES salt, Fisher, Hank's balanced salts; Sigma).

Table 3. Conditions and Set Points for the Dissolution Bath

\begin{tabular}{lc}
\hline Apparatus & USP II, paddles \\
\hline Paddle speed & $125 \mathrm{rpm}$ \\
Temperature & $37.0^{\circ} \mathrm{C}$ \\
Time points- & $5,10,15,20,30,45,60,75,90,120,150,180$ \\
SGF (min) & $5,10,15,2030,45,60,90,120,150,180,240$, \\
$\begin{array}{c}\text { Time points- } \\
\text { HEPES (min) }\end{array}$ & 300,360 \\
\hline
\end{tabular}

HEPES, 4-(2-hydroxyethyl)-1-piperazineethanesulfonic acid; SGF, simulated gastric fluid; USP, United States Pharmacopeia. 
At $T=0 \mathrm{~min}$ (baseline), $1.0 \mathrm{~mL}$ of CBD stock solution in methanol (equivalent to $40 \mathrm{mg} \mathrm{CBD}$ ) was spiked into separate vessels containing $500 \mathrm{~mL}$ of either SGF or HEPES buffer, and the paddles were started. At each time point, $1.0 \mathrm{~mL}$ of solution was withdrawn, and the amount of medium withdrawn from the test vessel was replaced with an equal volume of preheated medium. A maximum 3-h assessment time for SGF and 6-h assessment time for HEPES buffer exposure were chosen to nominally represent the maximal time of exposure of the substrate to the environment.
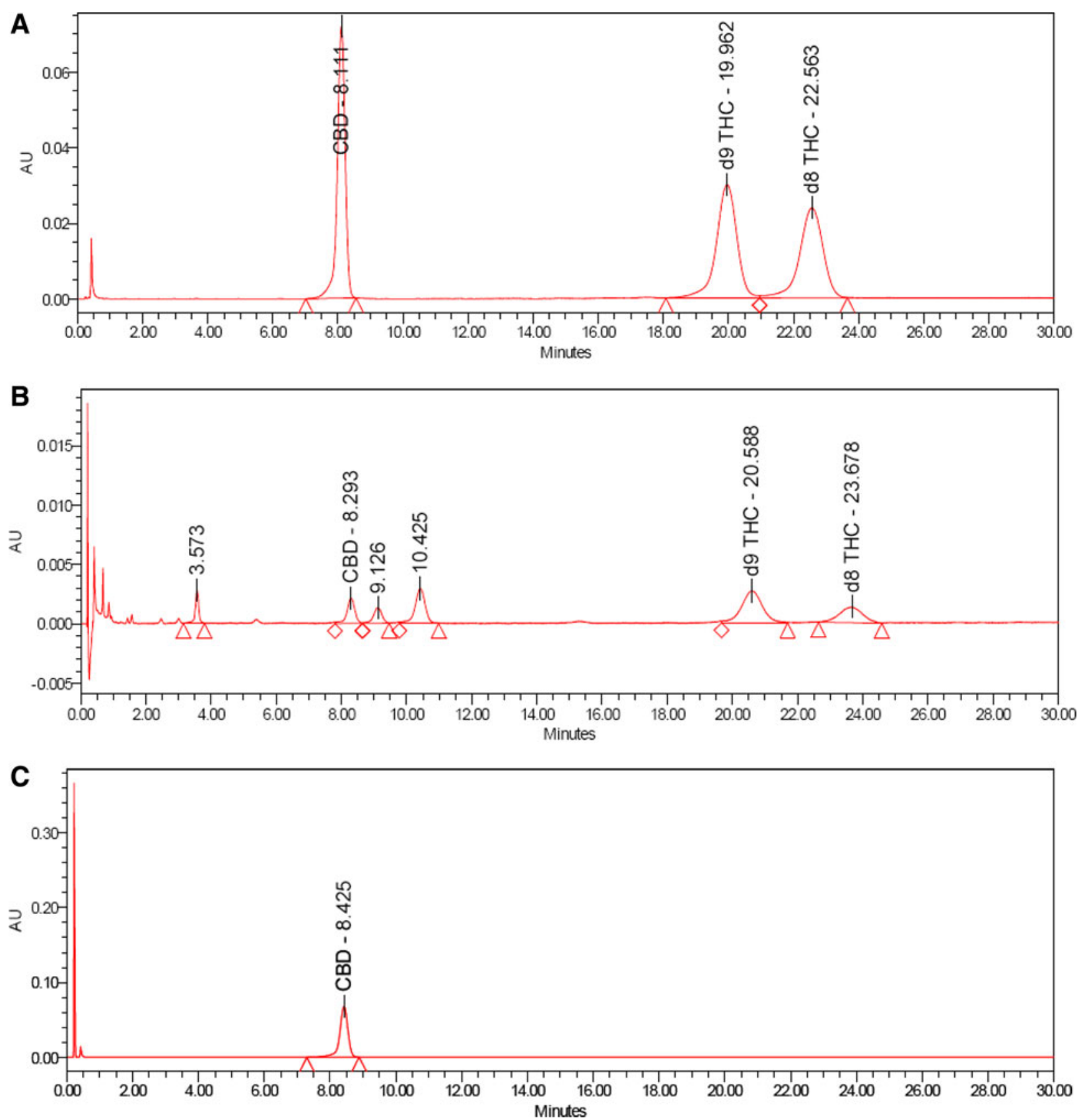

FIG. 2. UPLC-UV chromatograms in working standard (A), SGF at $75 \mathrm{~min}$ (B), HEPES at $360 \mathrm{~min}$ (C). UPLC, ultra-performance liquid chromatography; HEPES, 4-(2-hydroxyethyl)-1-piperazineethanesulfonic acid. 


\section{Results}

Cannabinoid assay

Good linearity by UPLC with UV detection was observed over the concentration range of 0.008 to $9.6 \mu \mathrm{g} / \mathrm{mL}$ for CBD $\left(R^{2}=1.0000, m=145,966, b=-837\right), \Delta^{8}$-THC $\left(R^{2}=1.0000, m=103,951, b=-1448\right)$, and $\Delta^{9}$-THC $\left(R^{2}=0.9999, m=125,280, b=-2735\right) ; y$-intercepts $<1 \%$ of the $100 \%$ response were obtained for each component. CBD, $\Delta^{8}$-THC, and $\Delta^{9}$-THC were identified by compar- ing the UV and mass spectra of the peaks in the SGF with the corresponding spectra of peaks in the working standard. No interference was observed in either the SGF or HEPES buffer. Excellent analyte separation was seen with the UPLC method (Figs. 2 [UV] and 3 [MS]). The mass spectra data confirmed the mass-tocharge ratio for CBD in SGF and physiological buffer and matched the CBD standard (Fig. 4). The mass spectra data confirmed the mass-to-charge ratio for
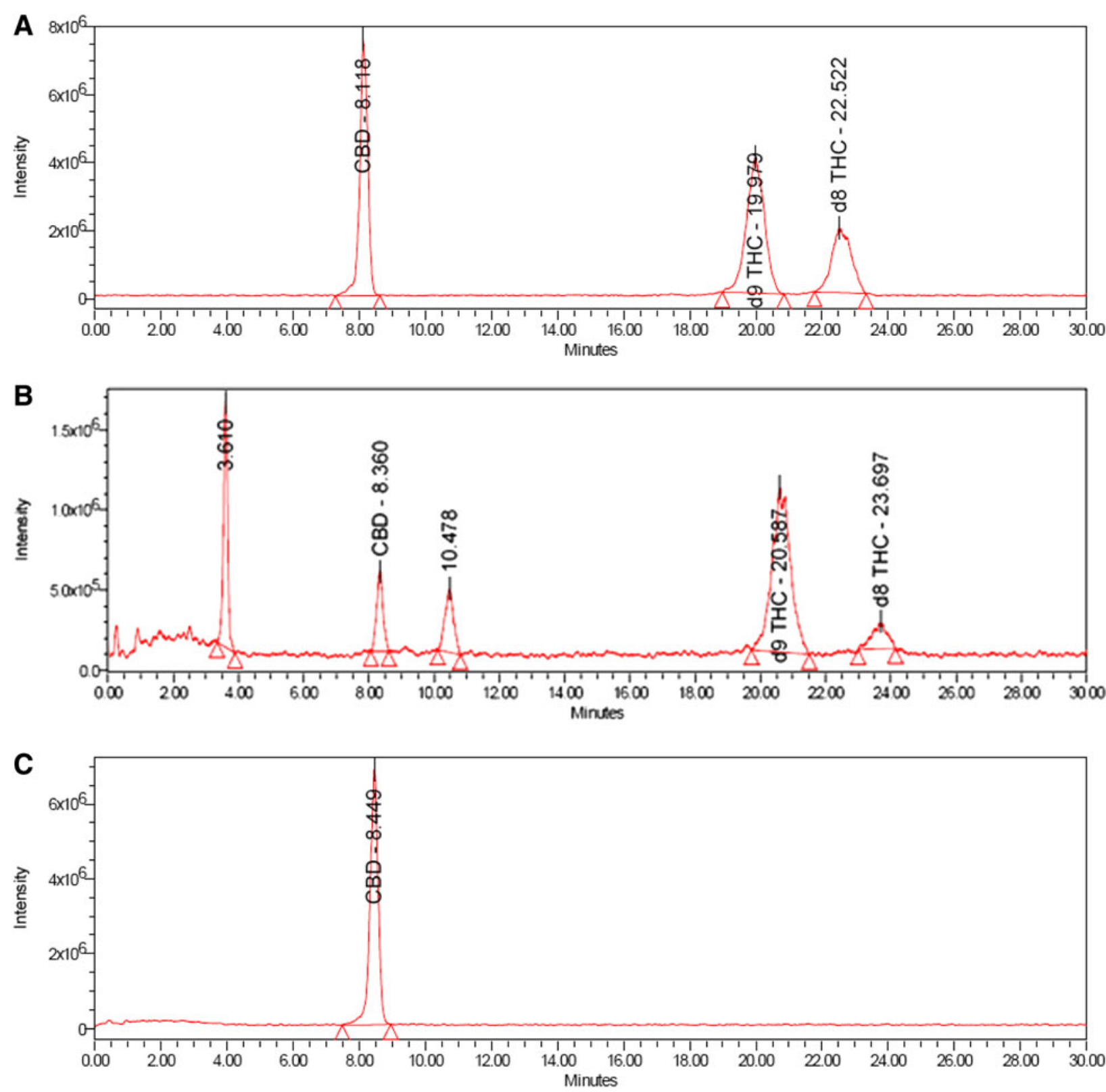

FIG. 3. Total ion chromatograms in working standard (A), SGF at 75 min (B), HEPES at $360 \mathrm{~min}$ (C). 

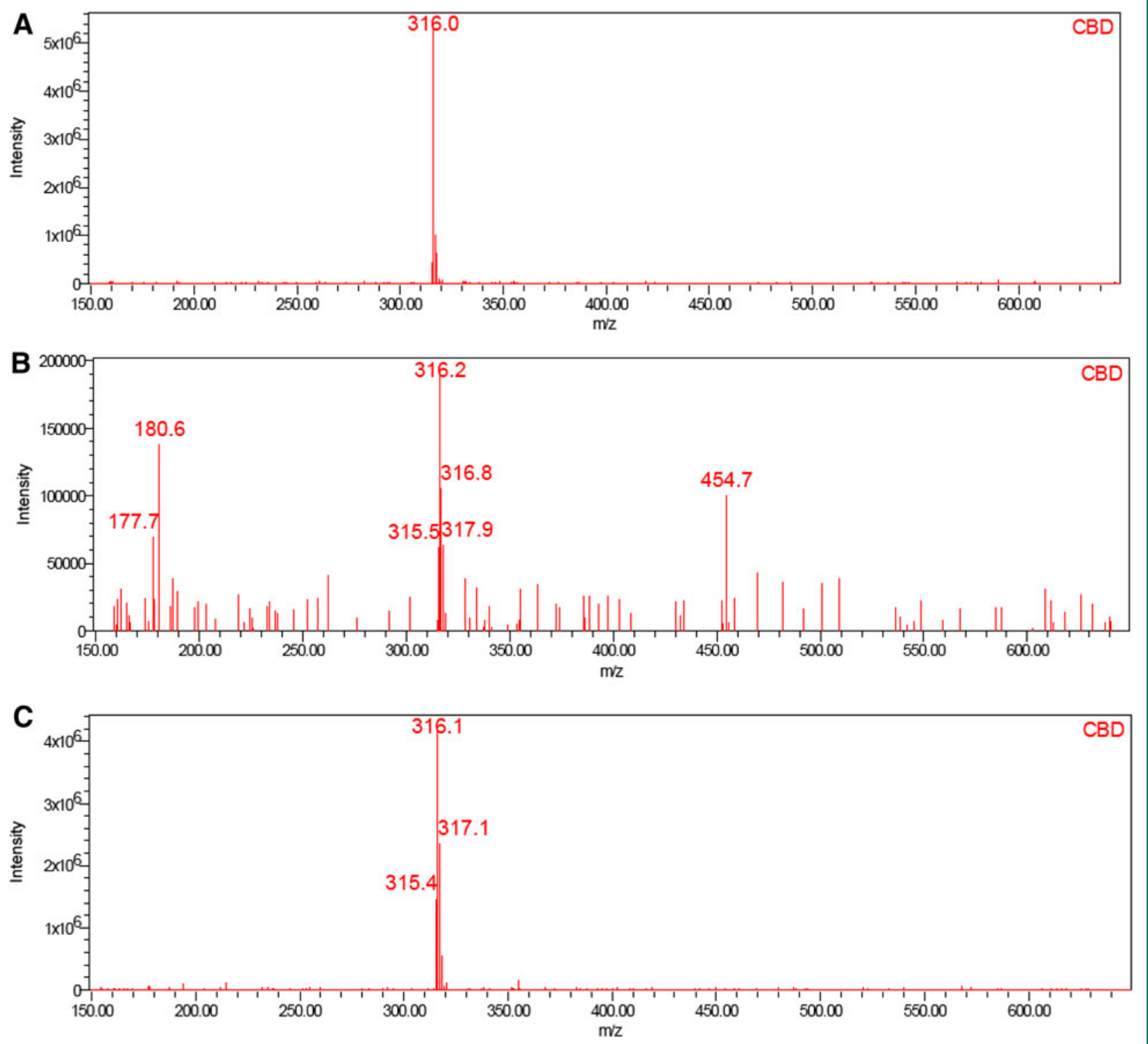

FIG. 4. Mass spectra of CBD in working standard (A), SGF at 75 min (B), HEPES at 360 min (C).

$\Delta^{8}$-THC and $\Delta^{9}$-THC formed in SGF and matched with the standards (Figs. 5 and 6). Table 4 provides a summary of the mass spectra data collected for CBD, $\Delta^{9}$ THC, and $\Delta^{8}$-THC standards and incubation samples.

Additional related substances at relative retention times (RRTs) of $0.44,1.09$, and 1.26 were also identified by UV in SGF samples (Fig. 2B). Two of the three peaks (RRTs 0.44 and 1.26) were detected in the multiple reaction monitoring (MRM) mode, confirming they are cannabinoids as the selected precursor and product ions were detected. The related substance peak at RRT 1.09 appears to be formed as a secondary product related to the RRT 0.44 product (Table 3 ) and is also most likely a related cannabinoid. The quantities of these related substances were calculated assuming a response factor of 1.0, equivalent to $\mathrm{CBD}$, to allow mass balance to be evaluated.

Incubation

In SGF, CBD degraded $~ 85 \%$ after $60 \mathrm{~min}$ and greater than $98 \%$ at $120 \mathrm{~min}$ (Table 5). The $\Delta^{9}$-THC: $\Delta^{8}$-THC ratio ranged from $\sim 1.25: 1$ to $1.5: 1$ over the course of the study period. CBD degradation and THC formation were very rapid (Fig. 7), and CBD consumption 



FIG. 5. Mass spectra of $\Delta^{9}$-THC in working standard (A) and SGF at 75 min (B). *A mass spectrum of $\Delta^{9}$-THC in HEPES at 360 minutes is not presented as there was none detected. THC, tetrahydrocannabinol.

demonstrated first-order kinetics, with a rate constant of $-0.031 \mathrm{~min}^{-1}\left(R^{2}=0.9933\right)$. Formation of THC isomers followed biphasic kinetics in which THC levels plateaued as $\mathrm{CBD}$ was consumed (Fig. 7). The THC levels were also impacted by secondary degradation to other related substances (Table 5). In HEPES buffer, no degradation of CBD to THC or other cannabinoids was observed over the 6-h duration of the study.

\section{Discussion}

This study demonstrated the acid-catalyzed cyclization of CBD to THC in SGF. CBD was degraded into the psychoactive cannabinoids $\Delta^{9}$-THC and $\Delta^{8}$-THC in SGF, and there was no evidence of CBD conversion to $\Delta^{8}$-THC or $\Delta^{9}$-THC in HEPES buffer. It was confirmed that the impurities were THC by favorably comparing the retention time of the sample peaks with those of the reference standards. The use of MS/MS detection in parallel with the UV detection verified the expected molecular weight of the com- pounds and provided direct confirmation that the peaks were THC.

The consistent CBD degradation in SGF led to a clear understanding of the kinetics of THC formation in an acidic environment, and the characterization of this rate enabled us to estimate the conversion of CBD to THC after oral dosing. Specifically, since CBD degradation demonstrated first-order kinetics, the formation of THC (and other related cannabinoids) can be conservatively estimated by using the inverse of this rate (i.e., $+0.031 \mathrm{~min}^{-1}$ ). The quantity of THC formed after oral administration of CBD-containing medications can thus be calculated-provided that the proportion of the CBD dose that would be soluble in the acidic gastric environment and thus "available" for degradation is also known. In a true physiological environment, this proportion depends on multiple factors, including (but not limited to) partitioning out of the lipid dosage form, enzyme activity, emulsification, and fasting state. Determining actual CBD solubility in gastric 

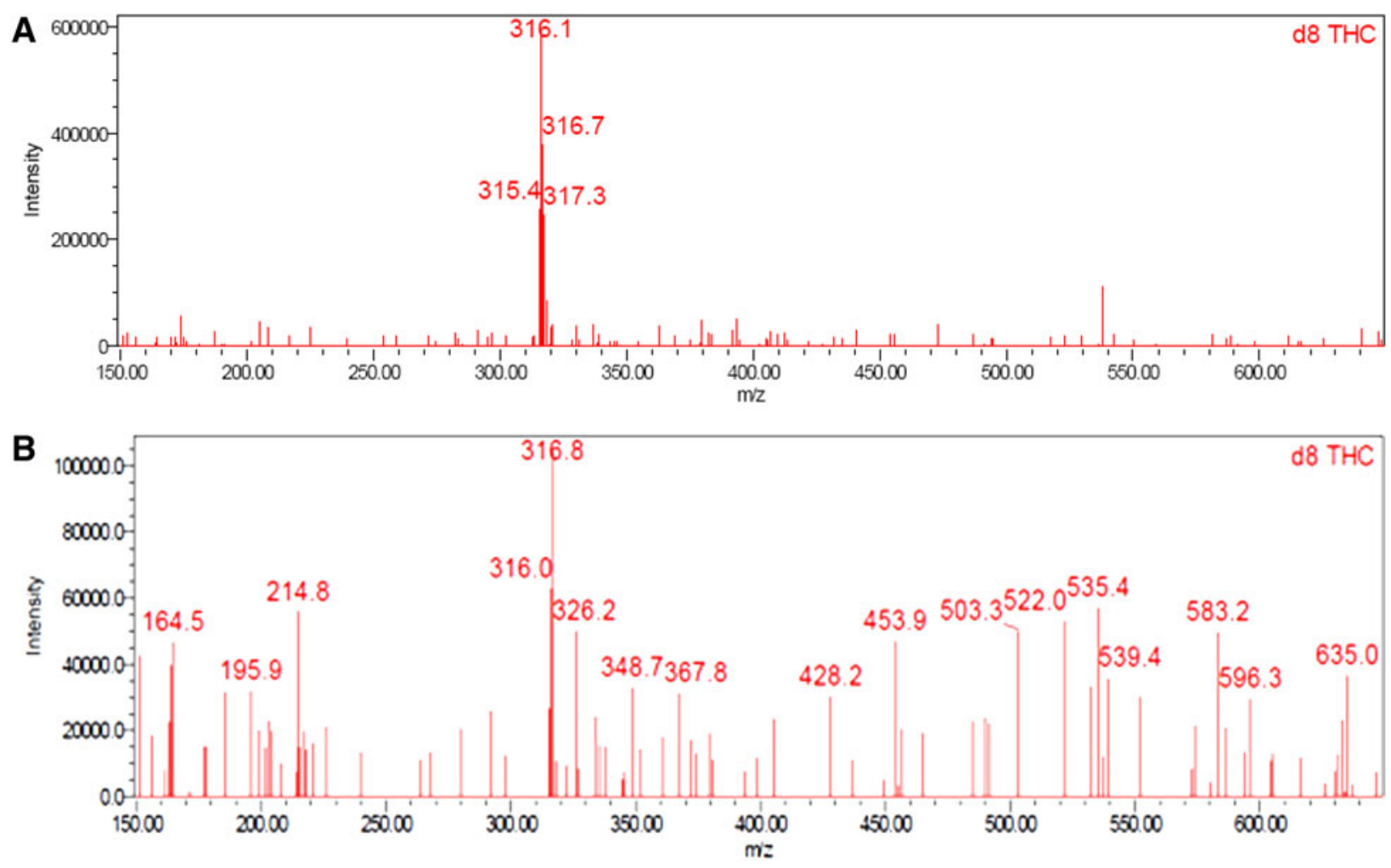

FIG. 6. Mass spectra of $\Delta^{8}$-THC in working standard (A) and SGF at 75 min (B). ${ }^{*} A$ mass spectrum of $\Delta^{8}$-THC in HEPES at 360 minutes is not presented as there was none detected.

fluid would require studies in human subjects. Based on our results, however, it is clear that at least some portion of an orally ingested dose of CBD will be soluble and degrade to THC.

We propose the following equation to describe THC exposure after a given time, where

THC exposure $(\mathrm{mg})=\mathrm{CBD}$ soluble in stomach $(\mathrm{mg})$

$$
\begin{aligned}
& \times \mathrm{K}_{\mathrm{THC}} \text { Formation }\left(\mathrm{min}^{-1}\right) \\
& \times \text { Time }(\min )
\end{aligned}
$$

In a patient treated with $700 \mathrm{mg}$ oral CBD formulated in a lipid environment (e.g., oil-based solution), even if just $1 \%$ of the CBD dose were soluble, total cannabinoid

Table 4. Summary of Mass Spectra Data

\begin{tabular}{lccc}
\hline Sample & CBD (m/z) & $\Delta^{8}$-THC (m/z) & $\Delta^{9}$-THC $(\mathrm{m} / \mathbf{z})$ \\
\hline Standard & 316.0 & 316.1 & 315.9 \\
75 min SGF & 316.2 & 316.8 & 315.9 \\
360 min HEPES buffer & 316.1 & $\mathrm{NA}^{\mathrm{a}}$ & $\mathrm{NA}^{\mathrm{a}}$ \\
\hline
\end{tabular}

${ }^{\mathrm{a}} \Delta^{8}$-THC and $\Delta^{9}$-THC were not detected in buffer sample. CBD, cannabidiol; THC, tetrahydrocannabinol. levels, primarily $\Delta^{9}$-THC and $\Delta^{8}$-THC with other degradation products, would be $6.5 \mathrm{mg}$ after $30 \mathrm{~min}$ and $13 \mathrm{mg}$ after $60 \mathrm{~min}$. Although the precise activity cannot be definitively determined until in vivo data are available, the central finding remains-significant levels of

Table 5. Degradation of CBD to THC and Related Substances in Simulated Gastric Fluid Containing $1 \%$ Sodium Dodecyl Sulfate

\begin{tabular}{lrrrrrrr}
\hline Min & CBD & $\Delta^{8}$-THC & $\Delta^{9}$-THC & $\begin{array}{c}\text { Total } \\
\text { THC }\end{array}$ & $\begin{array}{c}\text { Unknown } \\
\text { RRT 0.44 }\end{array}$ & $\begin{array}{c}\text { Unknown } \\
\text { RRT 1.09 }\end{array}$ & $\begin{array}{c}\text { Unknown } \\
\text { RRT 1.26 }\end{array}$ \\
\hline 5 & 34.6 & 2.0 & 2.5 & 4.5 & 2.4 & 0.0 & 0.0 \\
10 & 28.3 & 2.9 & 4.1 & 7.0 & 3.9 & 0.0 & 0.4 \\
15 & 23.3 & 3.7 & 5.0 & 8.7 & 4.7 & 0.0 & 0.8 \\
20 & 19.7 & 4.7 & 6.6 & 11.2 & 5.0 & 0.2 & 1.3 \\
30 & 14.1 & 6.6 & 9.7 & 16.2 & 4.8 & 0.6 & 2.3 \\
45 & 8.4 & 7.3 & 11.0 & 18.3 & 3.8 & 1.0 & 3.7 \\
60 & 5.2 & 8.1 & 12.2 & 20.3 & 2.6 & 1.5 & 4.5 \\
75 & 3.1 & 8.2 & 12.4 & 20.6 & 1.7 & 1.9 & 5.2 \\
90 & 1.9 & 8.7 & 12.4 & 21.1 & 1.2 & 2.3 & 5.6 \\
120 & 0.8 & 9.3 & 12.7 & 21.9 & 0.5 & 3.2 & 6.4 \\
150 & 0.3 & 8.7 & 11.1 & 19.8 & 0.2 & 3.7 & 6.5 \\
180 & 0.2 & 8.9 & 10.6 & 19.5 & 0.0 & 4.2 & 6.7 \\
\hline
\end{tabular}

Values are in mg (volume corrected). RRTs, relative retention times. 


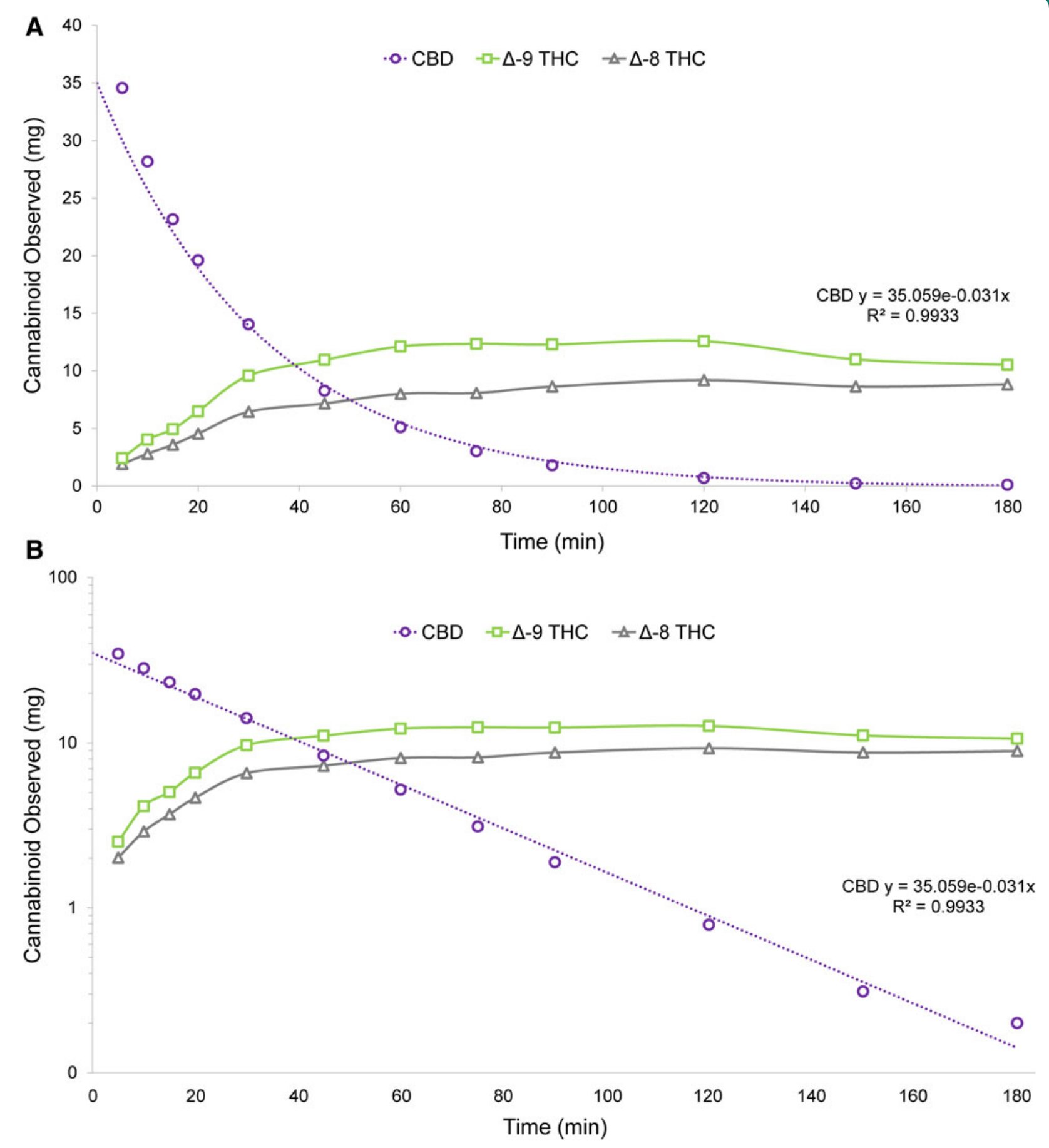

FIG. 7. CBD degradation in SGF-kinetics plotted on normal scale (A) and with CBD only on log scale (B).

psychoactive $\Delta^{9}$-THC, $\Delta^{8}$-THC, and other related compounds are formed when CBD is taken orally. With higher CBD doses, greater solubility, and/or longer gastric residence time, it is not difficult to envision scenarios in which $\Delta^{9}$-THC levels of $20-30 \mathrm{mg}$ or higher are reached (i.e., 1-1.5 times the maximum recommended daily dose) ${ }^{30}$

Our findings support the reproducibility of previous work. ${ }^{6,28,31-33}$ In the 1940s, before the structure of CBD had been established, Adams et al. observed that 
acidic conditions can convert CBD to cannabinoids that cause behavioral and physiological effects comparable to those seen with inhaled THC. ${ }^{31,32}$ In the 1960s, Gaoni and Mechoulam showed that various acidic reagents can degrade CBD and lead to the formation of $\Delta^{9}$ $\mathrm{THC}^{33}$ and $\Delta^{8}$-THC, ${ }^{6}$ the latter of which has been associated with physiological effects that are similar to, but less pronounced than, $\Delta^{9}$-THC (e.g., tachycardia and peak highs). ${ }^{34}$ More recent experiments have demonstrated that CBD can be converted to $\triangle^{9}$-THC in artificial gastric fluid, resulting in measurable pharmacological effects in murine models (e.g., catalepsy, hypothermia, sleep prolongation, and antinociception). ${ }^{28}$ This study extends these findings by showing the conversion rate of CBD to THC over 1 to $3 \mathrm{~h}$, a study period that approximates the duration of exposure in oral dosing situations and may have important implications for patients being treated with orally administered CBD.

If inhaled and human-metabolized CBD do not convert to $\Delta^{9}$-THC, as previous research suggests, ${ }^{30}$ and the trace amounts of THC in plant-extracted CBD medications cannot account for the high incidence of somnolence and fatigue in recent studies, ${ }^{26,27}$ the events may be partially explained by the sedating effects of antiepileptic drugs. ${ }^{35,36}$ However, these effects may also be a normal response to the psychoactive $\Delta^{9}$-THC and $\Delta^{8}$-THC released from the nonpsychoactive precursor CBD in the highly acidic gut milieu. Since studies of the effects of simulated gastric juice on CBD have shown that acid degradation also yields at least two hexahydrocannabinols that have been associated with catalepsy, hypothermia, sleep prolongation, and antinociception in mice, ${ }^{28}$ additive or synergistic activity at multiple cannabinoid receptors may be involved. Orally administered formulations of CBD, once thought devoid of the psychotropic side effects of THC, ${ }^{5,7,23}$ appear to convert in the gut by acid-catalyzed cyclization to clinically relevant concentrations of psychoactive cannabinoids, primarily $\Delta^{9}$-THC and $\Delta^{8}$-THC, that may affect clinical response and lead to adverse events.

Despite persistent challenges with dosing and administration, CBD-based therapies have a good safety profile $^{7,22}$ and a potential for efficacy in the treatment of a variety of medical conditions. The rapidly evolving sciences of drug delivery and cannabinoid pharmacology $^{1}$ may soon lead to breakthroughs that will improve access to the benefits of this pharmacological class of agents. In addition, current technologies, such as transdermal-based therapy, may be able to eliminate the potential for psychotropic effects due to this acid- catalyzed cyclization by delivering CBD through the skin and into the neutral, nonreactive environment of the systemic circulation.

\section{Conclusions}

Gastric fluid without enzymes converts $\mathrm{CBD}$ into the psychoactive components $\Delta^{9}$-THC and $\Delta^{8}$-THC, which suggests that the oral route of administration may increase the potential for psychomimetic adverse effects from CBD. Confirmation of THC formation was demonstrated by comparison of mass spectral analysis, mass identification, and retention time of THC in the SGF samples against authentic reference standards. The acid-catalyzed cyclization of CBD in SGF revealed firstorder kinetics of CBD degradation. This finding indicates that the acidic gastric environment during normal gastrointestinal transit may expose patients treated with oral CBD to levels of THC and other psychoactive cannabinoids that exceed the threshold for a physiological response. Delivery methods that decrease the potential for formation of psychoactive cannabinoids should be explored.

\section{Acknowledgments}

The authors thank Kiran Morishetti, $\mathrm{PhD}$, for optimizing and running the UPLC/MS sample analyses. This study was supported by Zynerba Pharmaceuticals, Inc. Medical writing services were provided by Christopher Caiazza.

\section{Author Disclosure Statement}

J.M. and B.L. were paid consultants, and T.S., C.O., and S.L.B. are employees, of Zynerba Pharmaceuticals. T.Y. has no competing financial interests.

\section{References}

1. Fine PG, Rosenfeld MJ. The endocannabinoid system, cannabinoids, and pain. Rambam Maimonides Med J. 2013;4:e0022.

2. Devinsky O, Cilio MR, Cross H, et al. Cannabidiol: pharmacology and potential therapeutic role in epilepsy and other neuropsychiatric disorders. Epilepsia. 2014;55:791-802.

3. El-Alfy AT, Ivey K, Robinson K, et al. Antidepressant-like effect of delta9tetrahydrocannabinol and other cannabinoids isolated from Cannabis sativa L. Pharmacol Biochem Behav. 2010;95:434-442.

4. Gaoni $Y$, Mechoulam R. Isolation, structure and partial synthesis of an active constituent of hashish. J Am Chem Soc. 1964;86:1646-1647.

5. Mechoulam R, Hanus L. Cannabidiol: an overview of some chemical and pharmacological aspects. Part I: chemical aspects. Chem Phys Lipids. 2002;121:35-43.

6. Gaoni Y, Mechoulam R. Concerning the isomerization of D1 to D6tetrahydrocannabinol. J Am Chem Soc. 1966;88:5673-5675.

7. Pertwee RG. The diverse CB1 and CB2 receptor pharmacology of three plant cannabinoids: $\Delta 9$-tetrahydrocannabinol, cannabidiol and $\Delta 9$ tetrahydrocannabivarin. Br J Pharmacol. 2008;153:199-215.

8. Abramovici H. Cannabis (marihuana, marijuana) and the cannabinoids. Available at www.hc-sc.gc.ca/dhp-mps/marihuana/med/infoprofeng.php (accessed February 16, 2016). 
9. Aggarwal SK. Cannabinergic pain medicine: a concise clinical primer and survey of randomized-controlled trial results. Clin J Pain. 2013;29:162-171.

10. Kraft B. Is there any clinically relevant cannabinoid-induced analgesia? Pharmacology. 2012;89:237-246.

11. Guindon J, Hohmann AG. The endocannabinoid system and pain. CNS Neurol Disord Drug Targets. 2009;8:403-421.

12. Mackie K. Signaling via CNS cannabinoid receptors. Mol Cell Endocrinol. 2008;286:S60-S65.

13. Maccarrone M, Gasperi V, Catani MV, et al. The endocannabinoid system and its relevance for nutrition. Annu Rev Nutr. 2010;30:423-440.

14. Serrano A, Parsons LH. Endocannabinoid influence in drug reinforcement dependence and addiction-related behaviors. Pharmacol Ther. 2011;132:215-241

15. Battista N, Di Tommaso M, Bari M, et al. The endocannabinoid system: an overview. Front Behav Neurosci. 2012;6:9.

16. Zhornitsky S, Potvin S. Cannabidiol in humans-the quest for therapeutic targets. Pharmaceuticals (Basel). 2012;5:529-552.

17. Robson P. Human studies of cannabinoids and medicinal cannabis. Handb Exp Pharmacol. 2005;168:719-756.

18. Jones NA, Hill AJ, Smith I, et al. Cannabidiol displays antiepileptiform and anti-seizure properties in vitro and in vivo. J Pharmacol Exp Ther. 2010;332:569-577.

19. Roser P, Vollenweider FX, Kawohl W. Potential antipsychotic properties of central cannabinoid (CB1) receptor antagonists. World J Biol Psychiatry. 2010;11:208-219.

20. Booz GW. Cannabidiol as an emergent therapeutic strategy for lessening the impact of inflammation on oxidative stress. Free Radic Biol Med. 2011;51:1054-1061.

21. Rock EM, Goodwin JM, Limebeer CL, et al. Interaction between nonpsychotropic cannabinoids in marihuana: effect of cannabigerol (CBG) on the anti-nausea or anti-emetic effects of cannabidiol (CBD) in rats and shrews. Psychopharmacology (Berl). 2011;215:505-512.

22. Cunha JM, Carlini EA, Pereira $A E$, et al. Chronic administration of cannabidiol to healthy volunteers and epileptic patients. Pharmacology. 1980;21:175-185.

23. Izzo AA, Borrelli F, Capasso R, et al. Non-psychotropic plant cannabinoids: new therapeutic opportunities from an ancient herb. Trends Pharmacol Sci. 2009;30:515-527.

24. Jones NA, Glyn SE, Akiyama S, et al. Cannabidiol exerts anti-convulsant effects in animal models of temporal lobe and partial seizures. Seizure. 2012;21:344-352.

25. Gupta S. Dr. Sanjay Gupta: It's time for a medical marijuana revolution. Available at www.cnn.com/2015/04/16/opinions/medical-marijuanarevolution-sanjay-gupta (accessed February 16, 2016).

26. Press CA, Knupp KG, Chapman KE. Parental reporting of response to ora cannabis extracts for treatment of refractory epilepsy. Epilepsy Behav. 2015;45:49-52.

27. Devinsky O, Sullivan J, Friedman D, et al. Epidiolex (cannabidiol) in treatment- resistant epilepsy. Poster presented at 67th Annual Meeting of the American Academy of Neurology, April 18-25, 2015, Washington, DC

28. Watanabe K, Itokawa Y, Yamaori S, et al. Conversion of cannabidiol to 49-tetrahydrocannabinol and related cannabinoids in artificial gastric juice, and their pharmacological effects in mice. Forensic Toxicol. 2007;25:16-21.

29. United States Pharmacopeia. The dissolution procedure: development and validation. Chapter 1092. Available at www.usp.org/sites/default/ files/usp_pdf/EN/gc_1092.pdf (accessed February 12, 2016).

30. Marinol ${ }^{\circledR}$ (dronabinol) capsules. Prescribing information. Unimed Pharmaceuticals, Inc.; Marietta, GA, 2006. Available at www.rxabbvie .com/pdf/marinol_PI.pdf (accessed February 16, 2016).

31. Adams R, Pease DC, Cain CK, et al. Structure of cannabidiol. VI. Isomerization of cannabidiol to tetrahydrocannabinol, a physiologically active product. Conversion of cannabidiol to cannabinol. J Am Chem Soc. 1940;62:2402-2405.

32. Adams R, Cain CK, McPhee WD, et al. Structure of cannabidiol. XII. Isomerization to tetrahydrocannabinols. J Am Chem Soc. 1941;63:2209-2213.

33. Gaoni Y, Mechoulam R. The isomerization of cannabidiol to tetrahydrocannabinols. Tetrahedron. 1966;22:1481-1488.

34. Gong H Jr., Tashkin DP, Simmons MS, et al. Acute and subacute bronchial effects of oral cannabinoids. Clin Pharmacol Ther. 1984;35 26-32.

35. Bansal D, Azad C, Kaur M, et al. Adverse effects of antiepileptic drugs in North Indian pediatric outpatients. Clin Neuropharmacol. 2013;36: 107-113.

36. Anderson M, Egunsola O, Cherrill J, et al. A prospective study of adverse drug reactions to antiepileptic drugs in children. BMJ Open. 2015;5:e008298.

Cite this article as: Merrick J, Lane B, Sebree T, Yaksh T, O'Neill C, Banks SL (2016) Identification of psychoactive degradants of cannabidiol in simulated gastric and physiological fluid, Cannabis and Cannabinoid Research 1:1, 102-112, DOI: 10.1089/can.2015.0004.

\begin{aligned} & \multicolumn{1}{c}{ Abbreviations Used } \\ & $\mathrm{CBD}=$ cannabidiol \\ & $\mathrm{ESI}=$ electrospray ionization \\ & $\mathrm{HEPES}=4-($-hydroxyethyl)-1-piperazineethanesulfonic acid \\ & $\mathrm{HSS}=$ high-strength silica \\ & $\mathrm{MRM}=$ multiple reaction monitoring \\ & $\mathrm{RRTS}=$ relative retention times \\ & $\mathrm{SDS}=$ sodium dodecyl sulfate \\ & $\mathrm{SGF}=$ simulated gastric fluid \\ & $\mathrm{THC}=$ tetrahydrocannabinol \\ & $\mathrm{TQD} \mathrm{MS} / \mathrm{MS}=$ tandem quadrupole mass spectrometry \\ & $\mathrm{UPLC}=$ ultra-performance liquid chromatography \\ & $\mathrm{USP}=$ United States Pharmacopeia \end{aligned}

\section{Publish in Cannabis and Cannabinoid Research}

Cannabis and Cannabinoid Research
- Immediate, unrestricted online access

- Rigorous peer review

- Compliance with open access mandates

- Authors retain copyright

- Highly indexed

- Targeted email marketing 